\title{
Age at natural menopause genetic risk score in relation to age at natural menopause and primary open-angle glaucoma in a US-based sample
}

\section{Citation}

Pasquale, L. R., H. Aschard, J. H. Kang, J. N. C. Bailey, S. Lindström, D. I. Chasman, W. G. Christen, et al. 2017. "Age at natural menopause genetic risk score in relation to age at natural menopause and primary open-angle glaucoma in a US-based sample." Menopause (New York, N.y.J 24 (2): 150-156. doi:10.1097/GME.0000000000000741. http://dx.doi.org/10.1097/ GME.0000000000000741.

\section{Published Version}

doi:10.1097/GME.0000000000000741

\section{Permanent link}

http://nrs.harvard.edu/urn-3:HUL.InstRepos:31731884

\section{Terms of Use}

This article was downloaded from Harvard University's DASH repository, and is made available under the terms and conditions applicable to Other Posted Material, as set forth at http:// nrs.harvard.edu/urn-3:HUL.InstRepos:dash.current.terms-of-use\#LAA

\section{Share Your Story}

The Harvard community has made this article openly available. Please share how this access benefits you. Submit a story. 


\title{
Age at natural menopause genetic risk score in relation to age at natural menopause and primary open-angle glaucoma in a US-based sample
}

Louis R. Pasquale, MD, ${ }^{1,2}$ Hugues Aschard, PhD, ${ }^{3}$ Jae H. Kang, ScD, ${ }^{2}$ Jessica N. Cooke Bailey, PhD, ${ }^{4,5}$ Sara Lindström, PhD, ${ }^{3}$ Daniel I. Chasman, PhD, ${ }^{6}$ William G. Christen, OD, PhD, ${ }^{6}$ R. Rand Allingham, MD, ${ }^{7}$ Allison Ashley-Koch, PhD, ${ }^{8}$ Richard K. Lee, MD, PhD, ${ }^{9}$ Sayoko E. Moroi, MD, PhD, ${ }^{10}$ Murray H. Brilliant, PhD, ${ }^{11}$ Gadi Wollstein, MD, ${ }^{12}$ Joel S. Schuman, MD, ${ }^{12}$ John Fingert, MD, PhD, ${ }^{13}$ Donald L. Budenz, MD, MPH, ${ }^{14}$ Tony Realini, MD, MPH, ${ }^{15}$ Terry Gaasterland, PhD, ${ }^{16}$ Douglas Gaasterland, MD, ${ }^{17}$ William K. Scott, PhD, ${ }^{18}$ Kuldev Singh, MD, MPH, ${ }^{19}$ Arthur J. Sit, MD, ${ }^{20}$ Robert P. IgoJr, PhD, ${ }^{4}$ Yeunjoo E. Song, PhD, ${ }^{4}$ Lisa Hark, PhD ${ }^{21}$ Robert Ritch, MD, ${ }^{22}$ Douglas J. Rhee, MD, ${ }^{23}$ Vikas Gulati, MD, ${ }^{24}$ Shane Havens, MD, ${ }^{24}$ Douglas Vollrath, MD, PhD, ${ }^{25}$ Donald J. Zack, MD, PhD, ${ }^{26}$ Felipe Medeiros, MD, ${ }^{27}$ Robert N. Weinreb, MD, ${ }^{27}$ Margaret A. Pericak-Vance, PhD, ${ }^{18}$ Yutao Liu, PhD, ${ }^{28}$ Peter Kraft, PhD, ${ }^{3}$ Julia E. Richards, PhD, ${ }^{10}$ Bernard A. Rosner, PhD, ${ }^{2,3}$ Michael A. Hauser, PhD, ${ }^{7,8}$ Jonathan L. Haines, PhD, ${ }^{4,5}$ and Janey L. Wiggs, MD, PhD ${ }^{I}$

\begin{abstract}
Objective: Several attributes of female reproductive history, including age at natural menopause (ANM), have been related to primary open-angle glaucoma (POAG). We assembled 18 previously reported common genetic variants that predict ANM to determine their association with ANM or POAG.

Methods: Using data from the Nurses' Health Study (7,143 women), we validated the ANM weighted genetic risk score in relation to self-reported ANM. Subsequently, to assess the relation with POAG, we used data from 2,160 female POAG cases and 29,110 controls in the National Eye Institute Glaucoma Human Genetics Collaboration Heritable Overall Operational Database (NEIGHBORHOOD), which consists of 8 datasets with imputed genotypes to 5.6+ million markers. Associations with POAG were assessed in each dataset, and site-specific results were meta-analyzed using the inverse weighted variance method.

Results: The genetic risk score was associated with self-reported ANM $\left(P=2.2 \times 10^{-77}\right)$ and predicted $4.8 \%$ of the variance in ANM. The ANM genetic risk score was not associated with POAG (Odds Ratio $(\mathrm{OR})=1.002 ; 95 \%$ Confidence Interval $(\mathrm{CI}): 0.998,1.007 ; P=0.28)$. No single genetic variant in the panel achieved nominal association with POAG $(P \geq 0.20)$. Compared to the middle 80 percent,
\end{abstract}

Received February 15, 2016; revised and accepted July 18, 2016.

From the ${ }^{1}$ Department of Ophthalmology, Massachusetts Eye and Ear Infirmary, ${ }^{2}$ Channing Division of Network Medicine, Brigham and Women's Hospital, ${ }^{3}$ Department of Epidemiology, Harvard T.H. Chan School of Public Health, Harvard Medical School, Boston, MA; ${ }^{4}$ Department of Epidemiology and Biostatistics, ${ }^{5}$ Institute of Computational Biology, Case Western Reserve University School of Medicine, Cleveland, OH; ${ }^{6}$ Division of Preventive Medicine, Brigham and Women's Hospital, Harvard Medical School, Boston, MA; ${ }^{7}$ Department of Ophthalmology, ${ }^{8}$ Department of Medicine, Duke University, Duke University Medical Center, Durham, NC; ${ }^{9}$ Bascom Palmer Eye Institute, University of Miami Miller School of Medicine, Miami, FL; ${ }^{10}$ Department of Ophthalmology and Visual Sciences, University of Michigan, Ann Arbor, MI; ${ }^{11}$ Center for Human Genetics, Marshfield Clinic Research Foundation, Marshfield, WI; ${ }^{12}$ Department of Ophthalmology, UPMC Eye Center, University of Pittsburgh, Pittsburgh, PA; ${ }^{13}$ Departments of Ophthalmology and Anatomy/Cell Biology, University of Iowa, College of Medicine, Iowa City, IO; ${ }^{4}$ Department of Ophthalmology, University of North Carolina, Chapel Hill, NC; ${ }^{15}$ Department of Ophthalmology, WVU Eye Institute, Morgantown, WV; ${ }^{16}$ Scripps Genome Center, University of California at San Diego, San Diego, CA; ${ }^{17}$ Emmes Corporation, Chevy Chase, MD; ${ }^{18}$ Hussman Institute for Human Genomics, University of Miami Miller School of Medicine, Miami, FL; ${ }^{19}$ Department of Ophthalmology, Stanford University, Palo Alto, CA; ${ }^{20}$ Department of Ophthalmology, Mayo Clinic, Rochester, MN; ${ }^{21}$ Wills Eye Institute,
Philadelphia, PA; ${ }^{22}$ Einhorn Clinical Research Center, New York Eye and Ear Infirmary of Mount Sinai, New York, NY; ${ }^{23}$ Department of Ophthalmology, Case Western Reserve University School of Medicine, Cleveland, OH; ${ }^{24}$ Department of Ophthalmology \& Visual Sciences, University of Nebraska Medical Center, Omaha, NE; ${ }^{25}$ Department of Genetics; Stanford University, Palo Alto, CA; ${ }^{26}$ Wilmer Eye Institute, Johns Hopkins University Hospital, Baltimore, MD; ${ }^{27}$ Department of Ophthalmology, Hamilton Eye Center; University of California at San Diego, San Diego, CA; and ${ }^{28}$ Department of Cellular Biology \& Anatomy, Augusta University, Augusta, GA.

Funding/support: Please see section at the end of the article.

Financial disclosure/conflicts of interest: Please see section at the end of the article.

Supplemental digital content is available for this article. Direct URL citations appear in the printed text and are provided in the HTML and PDF versions of this article on the journal's Website (www.menopause.org).

Address correspondence to: Louis R. Pasquale, MD, Mass Eye and Ear Infirmary, 243 Charles Street, Boston, MA 02115.

E-mail: Louis_Pasquale@meei.harvard.edu

This is an open access article distributed under the terms of the Creative Commons Attribution-Non Commercial-No Derivatives License 4.0 (CCBY-NC-ND), where it is permissible to download and share the work provided it is properly cited. The work cannot be changed in any way or used commercially without permission from the journal. 
there was also no association with the lowest $10^{\text {th }}$ percentile or highest $90^{\text {th }}$ percentile of genetic risk score with POAG $(\mathrm{OR}=0.75 ; 95 \% \mathrm{CI}: 0.47,1.21 ; P=0.23$ and $\mathrm{OR}=1.10 ; 95 \% \mathrm{CI}: 0.72,1.69 ; P=0.65$, respectively).

Conclusions: A genetic risk score predicting $4.8 \%$ of ANM variation was not related to POAG; thus, genetic determinants of ANM are unlikely to explain the previously reported association between the two phenotypes.

Key Words: Age at natural menopause - Genetic risk score - Primary open-angle glaucoma.

$\mathrm{P}$ rimary open-angle glaucoma (POAG) is an optic neuropathy strongly influenced by age and intraocular pressure that is a leading cause of irreversible blindness worldwide. ${ }^{1}$ Women carry a higher burden of visual disability related to POAG, presumably because they live longer than men. ${ }^{2}$ Although women are not necessarily more predisposed to POAG than men, attributes of female reproductive history linked to circulating estrogen levels are related to this disease. Later age at menarche, ${ }^{3,4}$ oral contraceptive use, ${ }^{4,5}$ early oophorectomy, ${ }^{6}$ and earlier age at menopause ${ }^{7,8}$ are associated with an increased risk of POAG. In contrast, later age at menopause ${ }^{9}$ and postmenopausal hormone use $^{10,11}$ were associated with reduced risk of POAG. Interestingly, post hoc analysis of a randomized clinical trial in the Women's Health Initiative revealed that postmenopausal estrogen use was associated with a modest $0.5 \mathrm{~mm} \mathrm{Hg}$ reduction in intraocular pressure, ${ }^{12}$ the only known modifiable risk factor for POAG.

Early menopause is associated with several nonocular diseases. ${ }^{13,14}$ With respect to glaucoma, in the Rotterdam Study, entering menopause before age 45 was associated with a 2.6-fold increased risk of open-angle glaucoma compared to entering menopause after age $50 .^{7}$ Among participants 65 years or older in the Nurses' Health Study, entering menopause at age 54 years or older was associated with a $47 \%$ reduced risk of high-tension POAG compared to entering menopause between the ages of 50 to $54 .{ }^{9}$ To better understand these results, we evaluated whether this relationship with POAG may be mediated by age at natural menopause (ANM) genetic biomarkers, because ANM is a highly heritable trait. ${ }^{15-18}$ Recently, several common genetic variants that predict menopause, a critical step in ovarian aging, have been identified. ${ }^{19}$ These variants do not overlap with the variants responsible for estrogen metabolism that we previously reported to be associated with POAG among women $^{20}$; nor do they overlap with known common gene variants for POAG. ${ }^{21}$ The nonoverlap between gene variants related to ANM and POAG could reflect the fact that genomewide significant loci for these traits represent variants with the most stringent $P$ values to reduce false discovery from multiple comparisons. ANM gene variants could represent novel biologically relevant POAG loci with associations that may have been obscured by the use of stringent $P$ values. Assembling ANM gene variants in a panel enhances the power to detect an overall association between ANM genetic variants and POAG while averting the multiple comparisons problem.
We validated the predictive ability of a previously published ANM genetic panel ${ }^{19}$ in relation to self-reported ANM in the Nurses Health Study and then determined the association with POAG in a large United States case-control dataset, composed of European-Americans referred to as the National Eye Institute Glaucoma Human Genetics Collaboration Heritable Overall Operational Database (NEIGHBORHOOD).

\section{METHODS}

\section{The nurses health study and the national eye institute glaucoma human collaboration heritable overall operational database}

The nurses health study (NHS) includes more than 121,000 female nurse participants who answered biennial questionnaires since cohort inception in 1976, including questions on reproductive status and various diseases such as glaucoma. ${ }^{22}$ Out of a subset of 11,522 women genotyped on three different high throughput platforms (various generation Illumina arrays specified below, the Illumina OmniExpress and the Affymetrix 6.0 arrays) for 11 disease endpoints, 7,143 had data on ANM and were included in the assessment of the relation between self-reported ANM and genetic risk score for ANM (discussed below). Women with no data on age at menopause or those with surgical menopause, history of pelvic radiation or menopause of unknown type were excluded. Data on menopause status in Nurses' Health Study has been previously validated. ${ }^{23}$

The national eye institute glaucoma human collaboration heritable overall operational database (NEIGHBORHOOD) dataset represents a genome-wide meta-analysis summary dataset from eight independent studies, including the Nurses' Health Study, with a total of 3,853 POAG cases and 33,480 controls of European ancestry from the United States. Details regarding the composition of the dataset used can be found in the study by Cooke Bailey et $\mathrm{al}^{24}$ and in Supplemental Table 1, Supplemental Digital Content 1, http:/links. lww.com/MENO/A180. A harmonized definition of POAG across these datasets consisted of the following features: open ocular anterior segment angles, pathologic cupping (cup-disc ratio $\geq 0.7$ in both eyes or inter-eye cup-disc ratio difference $\geq 0.2$ ), or at least one reliable visual field with deficits localizing to the optic nerve without secondary cause; although elevated intraocular pressure was not a criterion for POAG definition, if present, there had to be no secondary causes on anterior segment examination. We used the femaleonly meta-analyzed data in the NEIGHBORHOOD dataset from 2,160 POAG cases and 29,110 controls. This dataset 
partially overlapped with the NHS dataset used to assess the relation between ANM genetic risk score and self-reported ANM. From the Nurses' Health Study, we included 76 POAG cases and 2,488 controls who were genotyped on the Affymetrix 6.0 platform and 259 POAG cases and 1,367 controls who were genotyped on various generation Illumina platforms $(317 \mathrm{~K}, 550 \mathrm{~K}, 610 \mathrm{~K}$, and the $660 \mathrm{~W}$ arrays); controls who did not report an eye exam were not included in the POAG analyses.

\section{Formation of the age at natural menopause weighted genetic risk score}

The largest genome-wide meta-analysis of ANM included 53,403 women of European descent ${ }^{19}$ and identified 19 gene variants that were associated with ANM at the genome-wide significance level. Among the 19 gene variants associated with ANM reported by Stolk et al, ${ }^{19} 18$ were available in the Nurses Health Study and served as the basis for the ANM genetic risk score in our primary analysis. We also formed a larger genetic panel containing 44 common gene variants derived from a genome-wide scan of 69,360 women of European ancestry ${ }^{25}$ and present data related to this panel in Supplemental Table 2, Supplemental Digital Content 2, http://links.lww.com/MENO/A181, as these results did not differ from our primary analysis. To evaluate the relationship between ANM genetic risk score and self-reported ANM, genotypes were derived from the merged datasets in Nurses' Health Study described above. Details regarding how these datasets were merged are available in the supplemental material of the study by Cooke Bailey et al. ${ }^{24}$ Briefly, we created three platform-specific datasets (one each for earlier generation Illumina arrays, the Illumina OmniExpress array and the Affymetrix 6.0 array) and imputed the datasets using the 1,000 genomes phase I release. Each dataset underwent extensive quality control measures including analyses within and across the platforms to exclude duplicate samples and related individuals. To decrease false positives in imputation, we used only gene variants that overlap when combining all the datasets. The details of the quality control process used in imputing across platforms have been previously published. ${ }^{26}$ Gene variants that would create spurious associations and gene variants with poor imputation quality score at most 0.3 as determined by the $\mathrm{MaCH}$ software package designed to infer genotypes at un-typed loci were excluded.

For the NEIGHBORHOOD dataset, we used the metaanalysis results of POAG in women only. Details regarding the composition of the eight NEIGHBORHOOD datasets, genotyping, quality control measures, imputation and metaanalysis can be found in Cooke Bailey et al. ${ }^{24}$

\section{Statistical analyses}

To model ANM and POAG, we used multivariable linear regression and logistic regression, respectively. Individual gene variants were coded for the minor allele dosage associated with later-onset of ANM based on their imputation score with values ranging from zero for no alleles up to two, signifying two alleles. The ANM genetic risk score was defined as weighted based on the effect estimate of each gene variant or unweighted, where each gene variant carried equal weight.

In analyses for ANM, we used platform-specific results from the Nurses Health Study and meta-analyzed them with a computational tool called METAL, ${ }^{27}$ after ruling out heterogeneity with the Cochran Q-statistic. We adjusted for age in the year 1986, eigenvectors, and 11 disease endpoints including POAG (as genome-wide data in Nurses' Health Study was intended to assess other outcomes including coronary heart disease, type 2 diabetes, pancreatic cancer, kidney stones, glioma, colon cancer, gout, endometrial cancer, mammographic density, and ovarian cancer) in multivariate analyses.

In analyses for POAG, we used an inverse-variance weighted sum meta-analysis of individual SNP effect estimates from the female-only analysis, as proposed by Aschard. ${ }^{28}$ Let $\beta=\left(\beta_{\mathrm{G} 1}, \beta_{\mathrm{G} 2}, \ldots \beta_{\mathrm{Gm}}\right)$, a vector of elements of $\beta_{\mathrm{Gm}}$, which represents the reported effect of the $m$ th selected gene variant on POAG in the meta-analysis summary statistics and $\sigma_{\beta_{G_{i}}}^{2}$ represent the variance of each estimate. Under the assumption of independence between the gene variants (ie, assuming the gene variants' inter-correlations are negligible), $\chi^{2} G R S$, the chi-squared for the GRS effect on POAG, can be derived as:

$$
\chi^{2} G R S=\frac{\left(\sum_{m} \frac{w_{i} \times \beta_{G_{i}}}{\sigma_{\beta_{G_{i}}}^{2}}\right)^{2}}{\sum_{m} \frac{w_{i}^{2}}{\sigma_{\beta_{G_{i}}}^{2}}}
$$

We adjusted for age in years as a linear variable at DNA collection for POAG cases and controls as well as site-specific eigenvectors. To allow for differences in effect among the individuals with extreme values for the genetic risk score, we also considered a model to assess the relation between scores in the less than 10th percentile and more than 90th percentile relative to the middle $80 \%$ of genetic risk scores.

\section{RESULTS}

Mean ANM in the Nurses' Health Study sample was 50.6 years (standard deviation $=3.6 \mathrm{y}$ ). Age differences between cases and controls were adjusted for in genetic associations with POAG.

\section{Age at natural menopause genetic variants in relation to self-reported ANM in the nurses' health study}

Various studies have reported that between $2.5 \%$ to $4.1 \%$ of the reported total variance of ANM was explained by genes listed in Table $1 .{ }^{19} \mathrm{We}$ first tested each variant for association with self-reported ANM in the Nurses' Health Study using standard univariate linear regression. Almost all were nominally significant (16 of 18) and all showed positive correlation with reported ANM, in agreement with their previously reported effects (Table 1). ${ }^{19}$ The weighted gene risk score was 1.19 (Table 1) and the most significant gene locus was rs11668344 (TMEM150B; $\left.P=3.9 \times 10^{-20}\right)$. When all were 
simultaneously included in a multivariate model, these 18 gene loci jointly explained $4.8 \%$ of the variance of ANM in Nurses' Health Study, a slightly larger amount than reported in Stolk et al. ${ }^{19}$ The association between either the unweighted or weighted genetic risk scores and self-reported ANM was highly significant with $P$ values of $1.2 \times 10^{-61}$ and $2.2 \times 10^{-77}$, respectively. A 1.5-year difference in ANM between women at the 5 th percentile $(\mathrm{ANM}=49.0 \mathrm{y})$ and the 95 th percentile $(\mathrm{ANM}=50.5 \mathrm{y})$ of the genetic risk score was observed.

\section{Age at menopause gene variants in relation to primary open-angle glaucoma in the NEIGHBORHOOD dataset}

We tested the association between the 18 ANM gene variants and POAG in the NEIGHBORHOOD dataset using the summary statistics from the female-specific portion genome wide scan but no single locus showed even nominal associations with POAG $(P \geq 0.20$; Table 2$)$. We then tested the effect of either the unweighted or the weighted genetic risk score on POAG, first treating these scores as continuous variables, and subsequently, allowing for differences in effect among the individuals with extreme values. Neither the un-weighted genetic risk score (odds ratio $(\mathrm{OR})=1.013$; $95 \%$ confidence interval $(\mathrm{CI}): 0.990,1.036)$, nor the weighted score $(\mathrm{OR}=1.002 ; 95 \% \mathrm{CI}: 0.998,1.007)$ was associated with POAG. The lowest 10th and highest 90th percentiles of the weighted genetic risk scores were also not associated with POAG $(\mathrm{OR}=0.75 ; 95 \% \mathrm{CI}: 0.47,1.21 ; P=0.23$ and $\mathrm{OR}=1.10 ; 95 \% \mathrm{CI}: 0.72,1.69 ; P=0.65$, respectively). For the larger 44-member weighted and unweighted ANM genetic risk scores, we found identical null associations with POAG; the $P$ values were 0.42 and 0.87 , respectively (Supplemental Table 2, Supplemental Digital Content 2, http://links. lww.com/MENO/A181). Only a PIWIL1 locus (rs12824058) showed nominal association with POAG $(P=0.014)$ but the OR was in the opposite direction than expected (1.12) given the variant allele was associated with later ANM.

\section{DISCUSSION}

The ANM GRS panel was significantly predictive of selfreported ANM in the NHS, although it only explained $4.8 \%$ of the variance of ANM. This finding is consistent with previous reports in populations of varying ethnicities. ${ }^{29,30}$ Interestingly, although a few ANM genes are directly implicated in ovarian function, ${ }^{31,32}$ many are implicated in DNA repair mechanisms. ${ }^{25}$ Although our reported relation between ANM gene variants and self-reported ANM may be somewhat inflated due to the $8 \%$ overlap between Nurses' Health Study and Stolk et al ${ }^{19}$ they do serve to validate the biomarker panel as reflective of ANM.

We did not observe associations between the ANM genetic risk score and POAG, even when we considered the extremes of the score. Furthermore, none of the gene variants in the ANM panel were even marginally associated with POAG. It is unlikely that the sample was underpowered to find an association. With the large sample size of 2,160 cases and 29,110 controls in NEIGHBORHOOD, we had $80 \%$ power to detect an OR of 0.94 or lower for association between the ANM gene panel with POAG. Power dropped to $50 \%$ for an OR of 0.96 .

We leveraged high throughput genetic data in the largest POAG case-control set currently available in the form of a Mendelian randomization experiment. We used panels of genetic markers that predict ANM in relation to POAG. Agnostic search mechanisms reveal that many of these genes work through DNA repair mechanisms, involving enzymes such as HELQ and POLG, a DNA helicase and DNA polymerase respectively, both of which are strongly associated with

TABLE 1. ANM single nucleotide polymorphisms in relation to self-reported ANM in the Nurses' Health Study ( $n=7,143)$

\begin{tabular}{|c|c|c|c|c|c|c|}
\hline rs 4246511 & 1 & RHBDL2 & $\mathrm{T} / \mathrm{C}$ & 0.24 & 0.315 & $6.5 \times 10^{-6}$ \\
\hline rs1635501 & 1 & EXO1 & $\mathrm{T} / \mathrm{C}$ & 0.16 & 0.261 & $3.3 \times 10^{-5}$ \\
\hline rs10183486 & 2 & $T L K 1$ & $\mathrm{C} / \mathrm{T}$ & 0.20 & 0.161 & 0.012 \\
\hline rs4693089 & 4 & $H E L Q$ & $\mathrm{G} / \mathrm{A}$ & 0.23 & 0.296 & $1.7 \times 10^{-6}$ \\
\hline rs890835 & 5 & RNF44 & $\mathrm{A} / \mathrm{C}$ & 0.18 & 0.159 & 0.10 \\
\hline rs 2517388 & 8 & $A S H 2 L$ & $\mathrm{G} / \mathrm{T}$ & 0.26 & 0.254 & $2.5 \times 10^{-3}$ \\
\hline rs12294104 & 11 & near MPPED2 C11orf46 & $\mathrm{T} / \mathrm{C}$ & 0.23 & 0.159 & 0.054 \\
\hline rs2277339 & 12 & PRIMI & $\mathrm{T} / \mathrm{G}$ & 0.38 & 0.481 & $6.9 \times 10^{-6}$ \\
\hline rs 3736830 & 13 & KPNA3 & $\mathrm{C} / \mathrm{G}$ & 0.18 & 0.207 & 0.013 \\
\hline rs4886238 & 13 & $T D R D 3$ & $\mathrm{~A} / \mathrm{G}$ & 0.17 & 0.157 & 0.017 \\
\hline rs2307449 & 15 & $P O L G$ & $\mathrm{~T} / \mathrm{G}$ & 0.18 & 0.187 & 0.0030 \\
\hline rs10852344 & 16 & near TNFRSF17 RUNDC2A, GSPT1 & $\mathrm{C} / \mathrm{T}$ & 0.17 & 0.113 & 0.076 \\
\hline wGRS & & & & & 1.19 & $2.2 \times 10^{-77}$ \\
\hline
\end{tabular}

ANM, Age at natural menopause; GRS, Genetic Risk Score; SNP, single nucleotide polymorphism.

${ }^{a}$ GRS corresponds to the sum of all risk alleles; wGRS corresponds to the weighted sum of all risk alleles, where weights were defined as the beta coefficient of each SNP from Stolk et al. ${ }^{19}$ A0/A1 corresponds to the reference and coded allele, where the coded allele is associated later ANM. 
TABLE 2. Age at natural menopause single nucleotide polymorphisms in relation to primary open-angle glaucoma in the National Eye Institute Glaucoma Human Genetics Collaboration Heritable Overall Operational Database (2,160 cases and 29,111 controls)

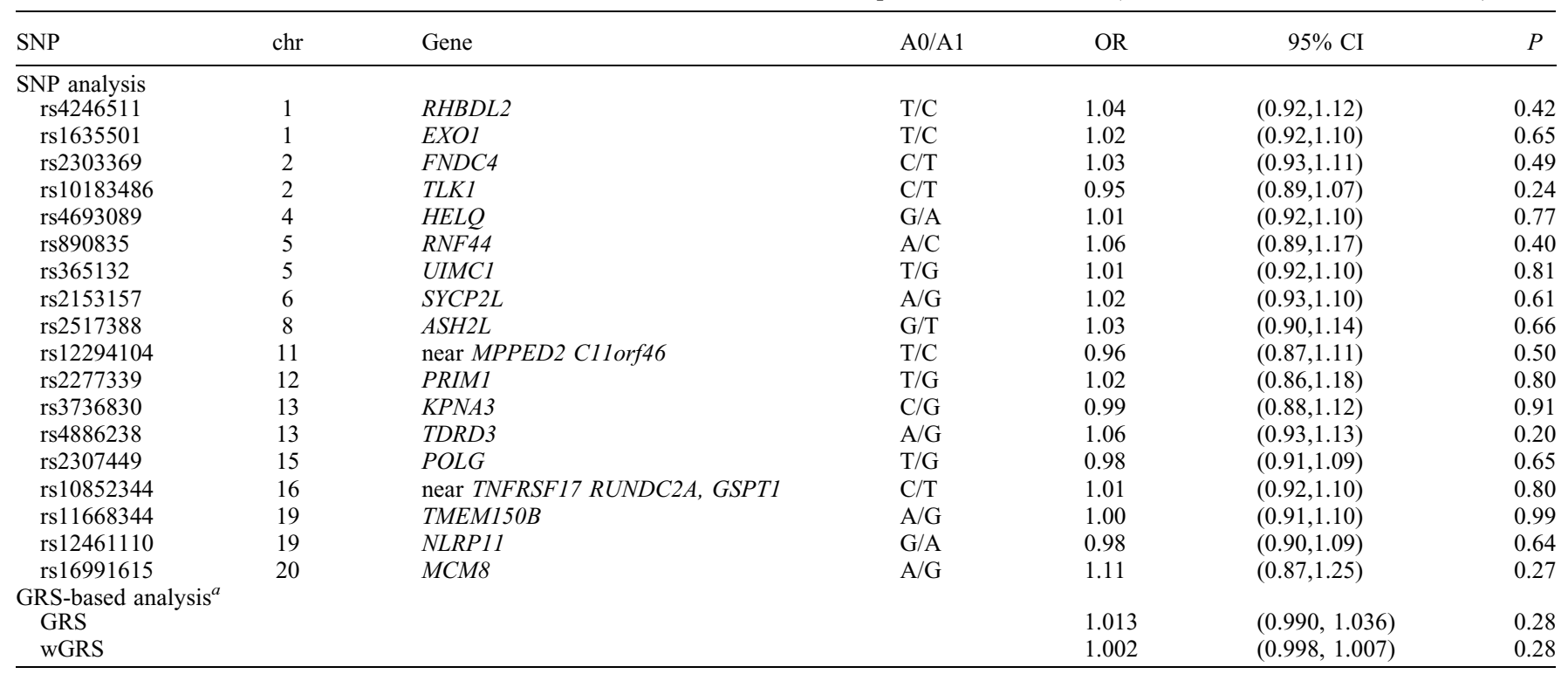

ANM, age at natural menopause; CI, confidence interval; GRS, Genetic Risk Score; OR, odds ratio; SNP, single nucleotide polymorphism. ${ }^{a}$ GRS corresponds to the sum of all risk alleles. wGRS corresponds to the weighted sum of all risk alleles, where weights were defined as the beta coefficient of each SNP from Stolk et al. ${ }^{19}$ A0/A1 corresponds to the reference and coded allele, where the coded allele is associated later ANM.

ANM (Table 1) but not associated with POAG (Table 2). Epidemiologic research supports the notion that declining estrogen levels are important in POAG pathogenesis. ${ }^{2-12}$ Our work suggests that for POAG, genetic ${ }^{20}$ and nongenetic exposures influencing estrogen levels ${ }^{5,9}$ may be more important than gene-based DNA repair mechanisms that may be more critical for other traits related to ANM such as breast cancer, ${ }^{25}$ where positive regulation of cell growth is observed.

$17-\beta$ estradiol is present in human trabecular meshwork cells ${ }^{33}$ that contribute to intraocular pressure generation and estrogen receptors are present on retinal ganglion cells ${ }^{34}$ that are selectively targeted for degeneration in POAG. Thus estrogenic input could be responsible for the lower IOP seen after estrogen replacement in postmenopausal women ${ }^{12}$ and the neuroprotective effects of estrogen observed in animal models of glaucoma. ${ }^{35,36}$ The definitive null association between ANM gene variants and POAG is critically important because it suggests these biomarkers, although related to ANM, are not viable targets to reduce the burden of POAG.

Although assembling genes into a panel serves to distinguish between true and false positive associations, in our instance no single gene in the panel achieved even nominal significance for association with POAG (Table 2). Even when we considered a larger 44-member ANM genetic panel in relation to POAG, our results were unequivocally null (Supplemental Table 2, Supplemental Digital Content 2, http://links.lww.com/MENO/A181), perhaps because the larger panel did not materially expand the biological pathway governing ANM much beyond DNA processing. ${ }^{25}$ Furthermore, the expanded panel only increased the variance in reported ANM explained by common gene variants from
$4.8 \%$ to $6 \%{ }^{25}$ Finally, considering POAG participants with either low or extremely high ANM genetic risk scores did not yield significant results. The difference in actual ANM was, however, relatively small for those at the extremes of the genetic risk score-women in the 5th percentile of the GRS had a mean ANM of 49 years, whereas those in the 95th percentile had a mean ANM of 50.5 years. Thus, although the genetic risk score is significantly predictive of ANM, even the extremes of this score may not be able to capture those with extreme actual ANM where associations with POAG have been observed. In the Rotterdam study, for example, ${ }^{7}$ the significant association with greater POAG risk was with early menopause (age $<45$ y) versus after age 50 years, which represented a difference of approximately 5 years in ANM. Although the discovery set for ANM genetic biomarkers was large $(\mathrm{n}=38,968),{ }^{19}$ even larger samples may be needed to discover missing heritability for ANM. There are likely hundreds of common genetic biomarkers that contribute to ANM and it may be worth re-examining the relation between genetic markers for ANM and POAG at a later date when more of these markers are known.

This study has some limitations. The findings are restricted to Caucasians and may not be applicable to other ethnic groups, as ANM does vary by ethnicity. ${ }^{37}$ Although the genetic panel for ANM explains only a small percentage of the variance in this trait, a larger panel explaining a greater percent of the variance was also not associated with POAG (Supplemental Table 2, Supplemental Digital Content 2, http://links.lww.com/MENO/A181). Furthermore, we previously showed that a genetic panel directly related to estrogen metabolism was associated with POAG in women but not in men. ${ }^{20}$ Finally, we did not account for other factors that 
might influence ANM such as smoking, body mass index, age at menarche, parity, and oral contraceptive use. ${ }^{37,38}$

\section{CONCLUSIONS}

Overall, although several attributes of female reproductive health are related to POAG, existing ANM gene variants, either individually or collectively are not related to glaucoma risk, indicating that genetic determinants of ANM are unlikely to explain the previously reported association between the two phenotypes.

Funding/support: The NIH/NEI R01EY022305 (J.L.W.) supports data collection and analysis for the National Eye Institute Glaucoma Human Genetics Collaboration Heritable Overall Operational Database (NEIGHBORHOOD) consortium. Support for collection of cases, controls, and analysis for individual datasets is as follows. Genotyping services for the predecessor National Eye Institute Glaucoma Human Genetics Collaboration (NEIGHBOR) study were provided by the Center for Inherited Disease Research (CIDR) and were supported by the National Eye Institute (NEI) through grant HG005259-01 (J.L.W.). In addition, CIDR is funded through a federal contract from the National Institutes of Health to The Johns Hopkins University—contract number HHSN268200782096C. Genotyping for the Mass Eye and Ear dataset and some Nurses Health Study (NHS) and Health Professionals Follow-up Study (HPFS) participants that formed the Glaucoma Genes and Environment (GLAUGEN) study was completed at the Broad Institute and supported by GENEVA project grant HG004728 (L.R.P.) and U01-HG004424 (Broad Institute). NIH/NHGRI U01 HG004446 (C Laurie) supported genotype data cleaning and analysis for the GLAUGEN study. The NEI through ARRA grants 3R01EY01587205S1 (J.L.W.) and 3R01EY019126-02S1 (M.A.H.) supported the collection and processing samples for the NEIGHBOR dataset. The funding for the collection of NEIGHBOR cases and controls was provided by NIH grants: EY015543 (R.R.A.), U02HG004608 (M.H.B.), HG006389 (M.H.B.), UL1TR000427 (M.H.B.), EY006827 (D.G.), HL73042, HL073389, EY13315 (M.A.H.); CA87969, CA49449, CA55075, EY009149 (PR Lichter), HG004608 (C McCarty), EY008208 (F.M.), EY015473 (L.R.P.), EY012118 (M.A.P.-V.), EY015682 (T.R.), EY011671 (J.E.R.), EY09580 (J.E.R.), EY013178 (J.S.S.), EY010886 (J.L.W.), EY009847 (J.L.W.), EY011008, EY144428 (K Zhang), EY144448 (K Zhang), EY18660 (K Zhang), and Research to Prevent Blindness (multiple institutions). The collection of Marshfield clinic cases and controls was supported by 1U02HG004608-01, 5U01HG006389-02, and NCATS/NIH grant UL1TR000427. In addition, some NHS/HPFS cases and controls and analysis of genome-wide data were supported by R01 CA131332 (RM Tamimi, I De Vivo), UM1 CA186107, UM1 CA167552, R01 CA49449, P01 CA87969. The Women's Genome Health Study (WGHS) is supported by HL043851 and HL080467 from the National Heart, Lung, and Blood Institute and CA047988 from the National Cancer Institute, the Donald W. Reynolds Foundation and the Foundation Leducq, with collaborative scientific support and funding for genotyping provided by Amgen. POAG case identification in Women's Genome Health Study was supported by 3RO1 EY015473-05S1 (LR Pasquale). Blood collection at New York Eye \& Ear Infirmary was supported by the New York Glaucoma Research Institute.
Financial disclosure/conflicts of interest: H.A., J.N.C.B., S.L., D.L.C., W.G.C., A.A.-K., S.E.M., D.G., M.H.B., G.W., R.P.I., Y.E.S., L.H., S.H., D.V., M.A.P.-V., and J.E.R. have no conflicts to declare. J.L.W., J.H.K., R.R.A., M.A.H., R.K.L., T.G., V.G., D.J.Z., Y.L., P.K., and B.A.R. sole disclosure is that they receive grant support from NIH. L.R.P. received grant support from NIH and has been a speaker for Allergan. He also served as a paid consultant to Novartis and to Bausch + Lomb. He has received support to travel to meetings by The Glaucoma Foundation (NYC), Glaukos and Aerie Pharmaceuticals. J.S.S. received grant support from NIH and is an inventor on a patent. J.F. received grant support from NIH and Regeneron. D.L.B. received consulting fees from Alcon Labs and travel support from New World Medical, Inc. He is also compensated for Data Safety Monitoring Board activity from Ivantis. He received grant support from New World Medical. T.R. received grant support from NIH and is a consultant for Alcon, Alimera, Bausch and Lomb, Reichert, Sensimed, and Inotek. W.K.S. holds a patent regarding the use of genetic data for risk assessment in age-related macula degeneration (Duke University). He received grant support from NIH, Florida Biomedical Research Program and the American Health Assistance Foundation. K.S. is a consultant to Alcon, Allergan, Santen, and Shire. A.H.S. serves as a consultant to Allergan, Alcon and Sensimed. He has received research support from NIH, Aerie Pharmaceuticals, and Glaukos. R.R. is on the advisory board of Isonic Inc, Intelon Optics and Xoma (US) LLC. He serves as consultant for Aeon Astron Europe B.V., Diopsyc, Inc, GLIA LLC, Gerson Lehrman Group, Guardian Health Sciences and Mobius Therapeutics. He is on the Board of Directors for International Eye Wellness Institute and receives royalties from Ocular Instruments. D.J.R. received research support from Merck, Allergan, Ivantis, and Glaukos. He is on the Scientific Advisory Board of Aerie and Transcend and is on the Data Safety Monitoring Board of Sanofi. F.M. received research support from Carl-Zeiss Meditec, Heidelberg Engineering, Allergan, Topcon, Reichert and Genentech. R.N.W. had a financial agreement or affiliation during the past year with the following commercial interests in the form of Consultant/Advisory Board: Alcon; Allergan; Bausch \& Lomb Incorporated; ForSight VISION5; and Valeant; Contracted Research: Genentech, Inc; and Quark. J.L.H. receives travel support and speaker honoraria from Novartis. He has received royalties from John Wiley and Sons and Athena Diagnostics. The NIH supports his research.

\section{REFERENCES}

1. Tham YC, Li X, Wong TY, et al. Global prevalence of glaucoma and projections of glaucoma burden through 2040: a systematic review and meta-analysis. Ophthalmology 2014;121:2081-2090.

2. Vajaranant TS, Pasquale LR. Estrogen deficiency accelerates aging of the optic nerve. Menopause 2012;19:942-947.

3. Lee AJ, Mitchell P, Rochtchina E, Healey PR. Female reproductive factors and open angle glaucoma: the Blue Mountains Eye Study. $\mathrm{Br} J$ Ophthalmol 2003;87:1324-1328.

4. Wang YE, Kakigi C, Barbosa D, et al. Oral contraceptive use and prevalence of self-reported glaucoma or ocular hypertension in the United States. Ophthalmology 2016;123:729-736.

5. Pasquale LR, Kang JH. Female reproductive factors and primary openangle glaucoma in the Nurses' Health Study. Eye (Lond) 2011;25:633-641.

6. Vajaranant TS, Grossardt BR, Maki PM, et al. Risk of glaucoma after early bilateral oophorectomy. Menopause 2014;21:391-398.

7. Hulsman CA, Westendorp IC, Ramrattan RS, et al. Is open-angle glaucoma associated with early menopause? The Rotterdam Study. Am $J$ Epidemiol 2001;154:138-144. 
8. Lam JS, Tay WT, Aung T, et al. Female reproductive factors and major eye diseases in Asian women-the Singapore Malay Eye Study. Ophthalmic Epidemiol 2014;21:92-98.

9. Pasquale LR, Rosner BA, Hankinson SE, Kang JH. Attributes of female reproductive aging and their relation to primary open-angle glaucoma: a prospective study. J Glaucoma 2007; 16:598-605.

10. Newman-Casey P, Talwar N, Nan B, et al. The association between postmenopausal hormone use and open-angle glaucoma. JAMA Ophthamol 2014;132:298-303.

11. Na KS, Jee DH, Han K, et al. The ocular benefits of estrogen replacement therapy: a population-based study in postmenopausal Korean women. PLoS One 2014;9:e106473.

12. Vajaranant TS, Maki PM, Pasquale LR, et al. Effects of hormone therapy on intraocular pressure in a randomized hormone trial: the Women's Health Initiative-Sight Exam Study. Am J Ophthalmol 2016;165: $115-124$

13. Atsma F, Bartelink ML, Grobbee DE, van der Schouw YT. Postmenopausal status and early menopause as independent risk factors for cardiovascular disease: a meta-analysis. Menopause 2006;13:265-279.

14. Gallagher JC. Effect of early menopause on bone mineral density and fractures. Menopause 2007;14(3 pt 2):567-571.

15. Snieder H, MacGregor AJ, Spector TD. Genes control the cessation of a woman's reproductive life: a twin study of hysterectomy and age at menopause. J Clin Endocrinol Metab 1998;83:1875-1880.

16. de Bruin JP, Bovenhuis H, van Noord PA, et al. The role of genetic factors in age at natural menopause. Hum Reprod 2001;16:2014-2018.

17. Murabito JM, Yang Q, Fox C, et al. Heritability of age at natural menopause in the Framingham Heart Study. J Clin Endocrinol Metab 2005;90:3427-3430.

18. Morris DH, Jones ME, Schoemaker MJ, et al. Familial concordance for age at natural menopause: results from the Breakthrough Generations Study. Menopause 2011;18:956-961.

19. Stolk L, Perry JR, Chasman DI, et al. Meta-analyses identify 13 loci associated with age at menopause and highlight DNA repair and immune pathways. Nat Genet 2012;44:260-268.

20. Pasquale LR, Loomis SJ, Weinreb RN, et al. Estrogen pathway polymorphisms in relation to primary open angle glaucoma: an analysis accounting for gender from the United States. Mol Vis 2013;19: 1471-1481.

21. Iglesias AI, Springelkamp H, Ramdas WD, et al. Genes, pathways, and animal models in primary open-angle glaucoma. Eye (Lond) 2015; 29:1285-1298.

22. Colditz GA, Hankinson SE. The Nurses' Health Study: lifestyle and health among women. Nat Rev Cancer 2005;5:388-396.
23. Colditz GA, Stampfer MJ, Willett WC, et al. Reproducibility and validity of self-reported menopausal status in a prospective cohort study. $\mathrm{Am} \mathrm{J}$ Epidemiol 1987;126:319-325.

24. Cooke Bailey JN, Loomis SJ, Kang JH, et al. Genome-wide association analysis identifies TXNRD2, ATXN2 and FOXC1 as novel susceptibility loci for primary open angle glaucoma. Nat Genet 2016;48:189-195.

25. Day FR, Ruth KS, Thompson DJ, et al. Large-scale genome analyses link reproductive aging to hypothalamic signaling, breast cancer susceptibility and BRCA1-mediated DNA repair. Nat Genet 2015;47:1294-1303.

26. Sinnott JA, Kraft P. Artifact due to differential error when cases and controls are imputed from different platforms. Hum Genet 2012;131: 111-119.

27. Willer CJ, Li Y, Abecasis GR. METAL: fast and efficient meta-analysis of genome-wide association scans. Bioinformatics 2010;26:2190-2191.

28. Aschard H. A perspective on interaction tests in genetic association studies [published online ahead of print July 7, 2016]. Genetic Epidemiol.

29. Shen C, Delahanty RJ, Gao YT, et al. Evaluating GWAS-identified SNPs for age at natural menopause among Chinese women. PLoS One 2013;8:e58766.

30. Carty CL, Spencer KL, Setiawan VW, et al. Replication of genetic loci for ages at menarche and menopause in the multi-ethnic Population Architecture using Genomics and Epidemiology (PAGE) study. Hum Reprod 2013;28:1695-1706.

31. Duncan AJ, Knight JA, Costello H, et al. POLG mutations and age at menopause. Hum Reprod 2012;27:2243-2244.

32. Schuh-Huerta SM, Johnson NA, Rosen MP, et al. Genetic markers of ovarian follicle number and menopause in women of multiple ethnicities. Hum Genet 2012;131:1709-1724.

33. Mookherjee S, Acharya M, Banerjee D, et al. Molecular basis for involvement of CYP1BI in MYOC upregulation and its potential implication in glaucoma pathogenesis. PLoS One 2012;7:e45077.

34. Munaut C, Lambert V, Nöel A, et al. Presence of oestrogen receptor type beta in human retina. Br J Ophthalmol 2001;85:877-882.

35. Zhou X, Li F, Ge J, et al. Retinal ganglion cell protection by 17-beta estradiol in a mouse model of inherited glaucoma. Dev Neurobiol 2007;67:603-616.

36. Prokai-Tatrai K, Xin H, Nguyen V, et al. 17ß-estradiol eye drops protect the retinal ganglion cell layer and preserve visual function in an in vivo model of glaucoma. Mol Pharm 2013;10:3253-3261.

37. Henderson KD, Bernstein L, Henderson B, et al. Predictors of the timing of natural menopause in the Multiethnic Cohort Study. Am J Epidemiol 2008;167:1287-1294.

38. Fleming LE, Levis S, LeBlanc WG, et al. Earlier age at menopause, work, and tobacco smoke exposure. Menopause 2008;15:1103-1108. 